\title{
Risk analysis of Volatile Organic Compounds using their indoor diffusion characteristics
}

\author{
T. Oyabu ${ }^{1}$, T. Higashino ${ }^{2} \&$ H. Nanto ${ }^{3}$ \\ ${ }^{1}$ Kanazawa Seiryo University, Japan \\ ${ }^{2}$ System Support Co., Ltd., Japan \\ ${ }^{3}$ Kanazawa Institute of Technology, Japan
}

\begin{abstract}
Diffusion characteristics of VOCs (Volatile Organic Compounds) were investigated using tin oxide gas sensors. The chemicals cause various kinds of symptoms to humans, for example sick building syndrome. In this study, the eight sensors were installed in a room. Four sensors were set in a vertical direction and the others on a plane surface. These sensors were of the same type. The VOC is put in a generation source, and the sensor output increases as the chemical diffuses. The output becomes higher as the concentration in the room becomes higher. The following chemicals were adopted as air pollution sources, namely formaldehyde, toluene and xylene. The sensor output is changed slightly even by a calm wind velocity. Therefore, the differential characteristic of sensor output was adopted and the noise component was removed as far as possible. A threshold value $v_{t h}$ to the characteristic was set up. It is understood that the examining chemical reaches the installed sensor point when the characteristic is in excess of the value. The speed reached was newly proposed using the threshold time $t_{t h}$ at which the output exceeded $v_{t h}$. The speed $s[\mathrm{~cm} / \mathrm{min}]$ is indicated using the distance $d$ and $t_{t h}$, namely $\mathrm{s}=d / t_{t h}$. The $d$ means the distance between the sensor position and the generation source. As a result, the speed for the sensor that is installed near a ceiling (the height is $260 \mathrm{~cm}$ from the floor) is fast, and it became obvious that the $s$ was larger to the smaller molecular chemical. The speed of formaldehyde for the sensor installed near the ceiling was $700[\mathrm{~cm} / \mathrm{min}]$ and the one for the sensor installed at the height of $100 \mathrm{~cm}$ from the floor was 370 [cm/min]. This is about double the difference in speed.
\end{abstract}

Keywords: VOC, gas diffusion, chemical sensor, sick house syndrome. 


\section{Introduction}

Various kinds of air polluting chemicals accumulate in indoor environments due to energy conservation measures and the following conditions and symptoms can arise: headaches, fatigue, asthma and sensing a drop in the level of concentration [1]. Patients who are critically ill have difficulty in leading a normal life and have to move to live in a clean-air area. These symptoms are called sick building, sick school or sick house syndrome. Nowadays, sick car syndrome, which occurs in a new car, is viewed with suspicion. In Japan, the Ministry of Health, Labor and Welfare, the Ministry of Land, Infrastructure and Transport, the Ministry of Education, Culture, Sports, Science and Technology and the Ministry of Environment stand at their guideline values in each stance. It is necessary to unify the management of those guideline values after accumulating many kinds of data concerning the symptoms. The main causative substances are VOCs (Volatile Organic Compounds), for example, formaldehyde, toluene and xylene [2]. The polluting level indoors has decreased compared with the late twentieth century. Atmospheric and water environments have deteriorated and many kinds of chemicals are also released from living wares. These chemicals affect humans.

Swelling of medical expenses as a result of sick building syndrome and the decline in labor productivity also figure strongly [3]. As a result, society is subjected to heavy damage by the syndrome. However, investigations concerning the diffusion and danger levels have not been carried out where there are VOCs as a contaminant source in an indoor environment [4-6]. In this study, the diffusion characteristics for formaldehyde, toluene and xylene, which are typical VOCs, were examined. As a result, it becomes obvious that larger molecular-weight chemicals diffuse at a slow rate and become more dangerous near the ceiling [7]. Air exhaust equipment at a higher position near a ceiling is required when these chemicals are present.

\section{Experimental}

The experiment was carried out in a space of $47.53 \mathrm{~m}^{3}(3.5 \times 1.53 \times 2.6 \mathrm{~m})$. The five support posts were set up in the room. Eight tin oxide gas sensors (Figaro TGS \#800) were installed to the posts. The sensors can be fixed at random heights, namely from floor to ceiling. The layout in the experimental room is indicated in Fig.1. Four sensors were installed at heights of 100, 150, 200 and $260 \mathrm{~cm}$ on the center post. The other four sensors were installed at the height of $260 \mathrm{~cm}$ (ceiling) on the other four posts respectively. These sensor outputs are described as ch 0 to 7 (\#0 to \#7). The sensor position and height are also indicated in the figure. The source of VOC outbreaks was set up at a distance of $40 \mathrm{~cm}$ from the center post. The output signals (ch0-7) from these sensors are input into a data logger through an A-D converter. After that, the data are input into the mobile computer and stored in the memory. The sampling interval is 0.1 seconds. The photograph of the installed sensor is incorporated in Fig.2. The heights of all of the sensors are as follows. The ch0 to 3 are installed on the center post. 


$$
\begin{aligned}
& \text { ch0 : } 100 \mathrm{~cm} \\
& \text { ch1 : } 150 \mathrm{~cm} \\
& \text { ch2 : } 200 \mathrm{~cm} \\
& \text { ch3-7 }: 260 \mathrm{~cm}
\end{aligned}
$$

The sealed case of $50 \mathrm{ml}$, in which a testing chemical $(10 \mathrm{ml})$ was contained, was opened. The case is the generating source and the height is $1 \mathrm{~cm}$ from the floor. The sensor outputs were examined. In this experiment, solutions of formaldehyde $(\mathrm{HCHO}$, molecular weight $30.03 \mathrm{~g} / \mathrm{mol})$, toluene $\left(\mathrm{C}_{7} \mathrm{H}_{8}\right.$, molecular weight $92.14 \mathrm{~g} / \mathrm{mol})$ and xylene $\left(\mathrm{C}_{8} \mathrm{H}_{10}\right.$ molecular weight $\left.106.17 \mathrm{~g} / \mathrm{mol}\right)$ are adopted as polluting sources. These polluting chemicals commonly evaporate from various kinds of furniture. The air exchanging rate was about $0.5 /$ hour.

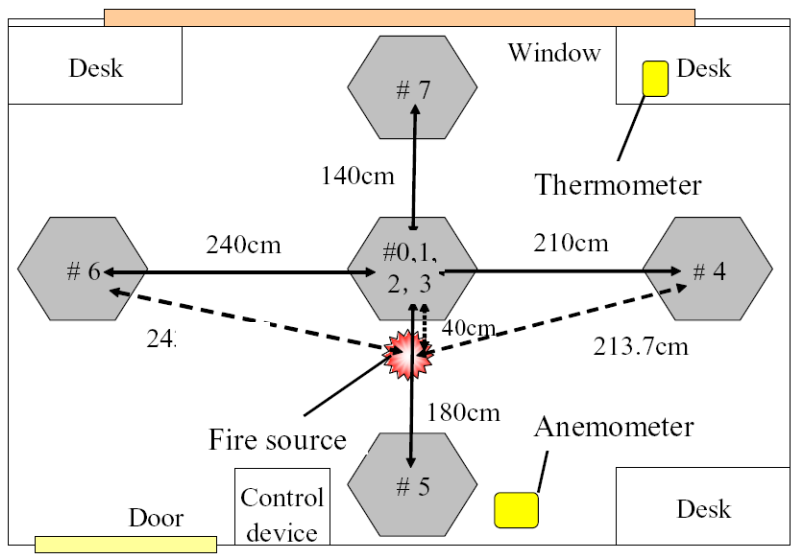

$\# 3,4,5,6,7=260 \mathrm{~cm}, \# 0=100 \mathrm{~cm}, \# 1=150 \mathrm{~cm}, \# 2=200 \mathrm{~cm}$

Figure 1: $\quad$ Layout of experimental room.

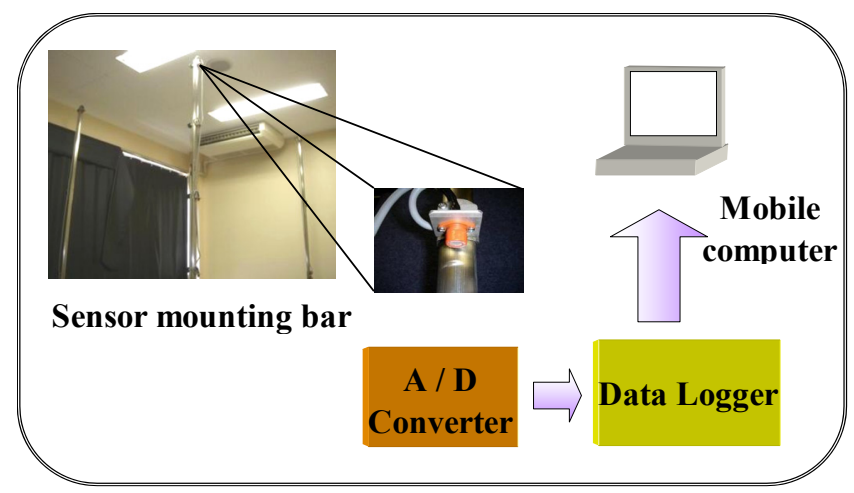

Figure 2: $\quad$ Outline of sensing system. 


\section{Experimental result}

\subsection{Sensor output characteristic according to VOC diffusion}

In Japan, the amount of VOC production is now over several million tons and it is routinely emitted into the atmosphere. The concentration of the gases is controlled in an indoor environment by a regulation value and the accumulation of VOCs in a building is now widely recognized. The main chemicals are toluene and xylene. The concentration of these chemicals decreases remarkably when a window is opened and balances with the levels outside. Therefore, it is important to grasp the inside concentration when the room is closed.

In this study, each chemical solution of $10 \mathrm{ml}$ was put into the sealed case of $50 \mathrm{ml}$ and it was opened at the start of the experiment. The VOC begins to evaporate at the start. The gas sensor has no gas selectivity, namely it has sensitivity to many reducing gases but the sensitivity level for each chemical is different respectively. It has a tendency to have larger sensitivity to large molecular-weight chemicals. The sensor output fluctuates even in the calm condition in the room. Therefore, new value $V_{i s}$ is introduced, which is derived by the summation of sensor output every 0.1 seconds. In this study, $V_{i s}$ for five seconds is adopted, namely fifty outputs are added in the value. The example of the characteristic is shown in Fig.3. The upper part of the figure shows the characteristics from the sensors that are installed on the center post, namely ch03. The lower part of the figure shows the sensor outputs (ch4-7) that are installed at the height of ceiling on the other four posts. The sealed case was opened and formaldehyde was emitted one hour after the start of the experiment. The summation value $V_{i s}$ increased remarkably in an hour after its opening and there was a tendency that the characteristic was saturated in the next couple of hours. In the characteristics, there are no large differences among sensor heights, for example ch0-3. There is large position-related difference, namely in ch4-7.

\subsection{Differential characteristic}

It is also considered that the VOC concentration increases suddenly by the rapid increase of room temperature in an indoor environment. It is necessary to detect the exact time at which the concentration increased and to inform someone about the time. The time when a chemical emits has to be derived from the characteristic. The differential characteristic $V_{\text {diff }}$ for $V_{\text {is }}$ characteristic was newly introduced. The system can identify whether some sort of event (VOC generation) occurs or not using the $V_{\text {diff }}$ characteristic. The differential characteristics $V_{\text {diff }}$ for Fig.3 are indicated in Fig.4. The characteristic is obtained by introducing the difference between each plot. An event can be recognized using the characteristic, because whole sensor characteristics $V_{\text {diff }}$ remarkably fluctuate at the generation of VOC. The $V_{\text {diff }}$ characteristic is broad after an event happened. The gas sensor characteristic contains some noise components and the original data have to be processed to recognize the events. The process idea is important in this field. In Fig.4, the upper part of the figure shows the 
characteristics for ch0-3 and the lower part of the figure shows the ones for ch47. A threshold value $V_{t h}$ is adopted for $V_{\text {diff }}$ characteristics to identify an event. The system regards the condition as an event occurring when the $V_{\text {diff }}$ exceeds the value $V_{t h}$. The detailed characteristic when an event occurs is shown in Fig.5. Figure 5(a) shows the characteristics for ch0-3 and 5(b) shows the characteristics for ch4-7. The features differ in the characteristics, namely the modified characteristic and identifying time differ in sensor height and position. The event occurred at $10 \mathrm{~min}$ in Fig.5. Many experiments were carried out and the system adopted the value $V_{t h}=0.3[\mathrm{~V} / \mathrm{sec}]$ time to identify the event in ch2, 4 and 6 . The $t_{t h}$ are long in these three channels.

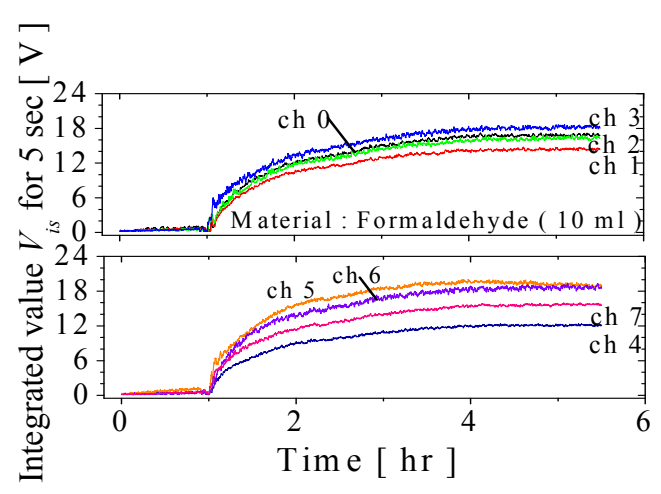

Figure 3: $\quad$ Sensor characteristics $V_{i s}$ for formaldehyde.

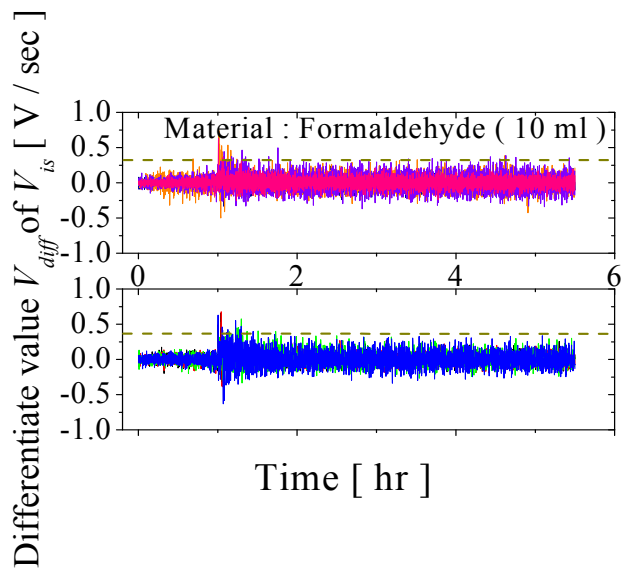

Figure 4: Differential characteristics $V_{\text {diff }}$ for Fig.3. 


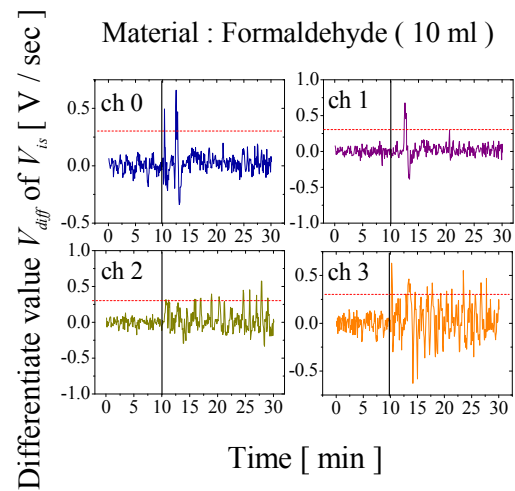

(a)

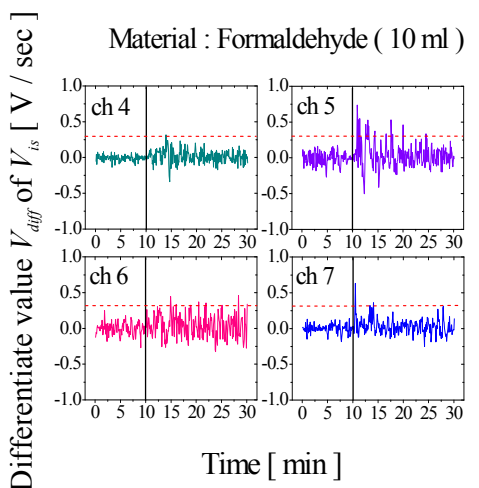

(b)

Figure 5: In-depth characteristics of $V_{\text {diff }}$ for each sensor: (a) $\operatorname{ch} 0$ to $\operatorname{ch} 3$ and (b) ch4 to ch7.

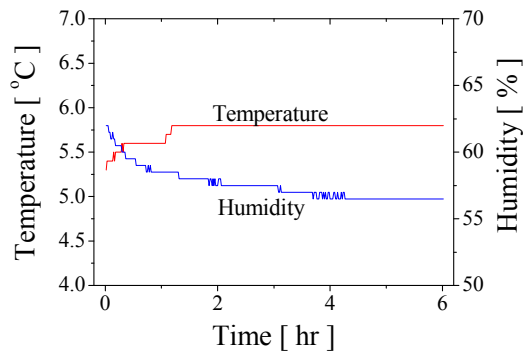

Figure 6: Temperature and humidity characteristics during the experiment.

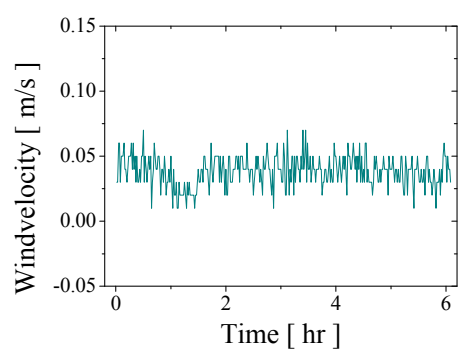

Figure 7: Air-volume characteristic during experiment.

This identifies as an event (VOC) occurring when $V_{t h}>=0.3[\mathrm{~V} / \mathrm{sec}]$ and the time is named as $t_{t h}$. The $t_{t h}$ means the time that a VOC reaches the sensor. A VOC does not diffuse homogeneously even in a calm air volume. The $V_{\text {diff }}$ may not exceed $V_{t h}$ in some positions. In the figure, the $V_{\text {diff }}$ for all sensors exceeds the $V_{t h}$ in several minutes.

The room temperature and humidity characteristics during the experiment are indicated in Fig.6. The experiment was carried out in winter. The temperature was low and the range was 5.5 to 6.0 degree C. The humidity was 55 to 60 $[\% \mathrm{RH}]$. These values became nearly steady-states, namely 5.75 degree $\mathrm{C}$ and $56.5[\% \mathrm{RH}]$. The temperature and humidity have a negative correlation ship. The air-volume sensor characteristic is presented in Fig.7. It denotes from 0.0 to $0.075[\mathrm{~m} / \mathrm{s}]$ during the experiment. The characteristic is a micro flow. However, 
the flow affects the VOC diffusion to some degree. The time $\left(t_{t h}\right)$ for each channel during the formaldehyde diffusion experiment, at which an event occurred, was derived.

The result is exhibited in Fig.8. The $t_{t h}$ for ch0-5 were smaller than 13 min. The ch3-5 detected the event in five minutes. The ch6 and 7 detected it more than 25 min later. It was considered that there was a light air flow in the lower right direction in the results shown in Fig.1. The specific gravity of formaldehyde is lighter-than-air and the value is 0.815 . It moves easily with an air stream and diffuses to the upper side.

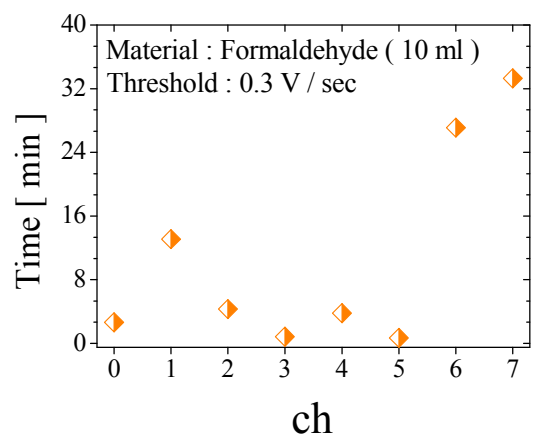

Figure 8: The time $t_{t h}$ at which $V_{\text {diff }}$ exceeds the threshold value $V_{t h}$. The horizontal axis shows the sensor number.

\subsection{VOC propagation speed}

New coefficient " $s$ " is now introduced. The linear distance between the VOC generating source and each sensor is named as $c_{t h} . s=c_{t h} / t_{t h}[\mathrm{~cm} / \mathrm{min}]$. The $s$ means a certain type of speed at which a VOC reaches the sensor position. The $s$ characteristics to formaldehyde are exhibited in Fig.9. The characteristics for ch0, 3, 5 and 7 are higher. It is understood that the value at the ceiling position just above the polluting source is the quickest. The concentration at the ceiling is the highest and this phenomenon is the same as the chimney effect. The $s$ characteristic for toluene is shown in Fig.10. The speeds $s$ for ch0, 2, 3, 5 and 6 are fast. The $s$ value for ch1 $(150 \mathrm{~cm}$ height from floor) is small and it is the same as the one for formaldehyde. The risks for small babies and people during sleep hours are higher. It is necessary to exhaust toluene at high and low heights. The characteristic for xylene is indicated in Fig.11. The ch4 value is small compared with the other channel numbers. The $s$-values for $\operatorname{ch} 0$ and 3 to three chemicals are shown in Fig.12. The sensors of the channels are installed on the center post, namely ch0 is at the lowest height and ch3 is at the greatest height. In the figure, the horizontal axis is the molecular weight, but it means only the weight order. The $s$-values of ch0 to the three chemicals differ little with each other. The value of ch3 at the ceiling becomes smaller as the weight becomes 
10 Environmental Health Risk V

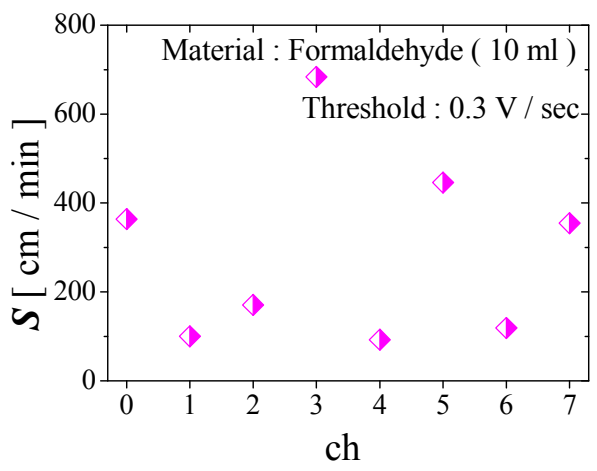

Figure 9: $\quad$ The $s$-values of each sensor for formaldehyde. The value shown is $c_{t h} / t_{t h}[\mathrm{~cm} / \mathrm{min}]$.

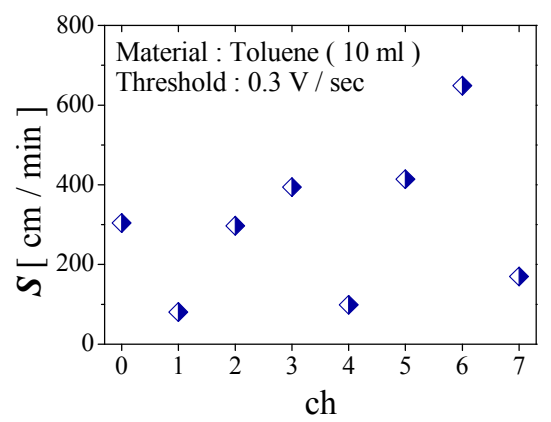

Figure 10: The $s$-values of each sensor for toluene.

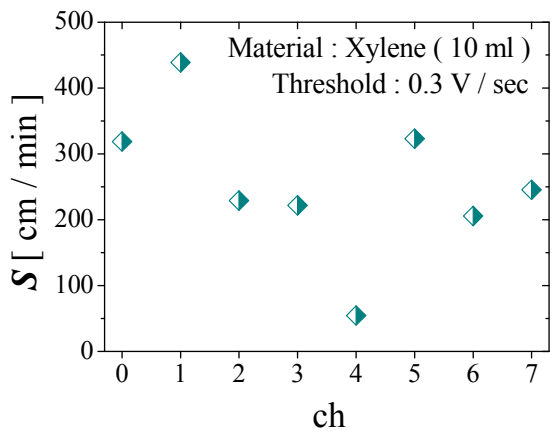

Figure 11: The $s$-values of each sensor for xylene. 


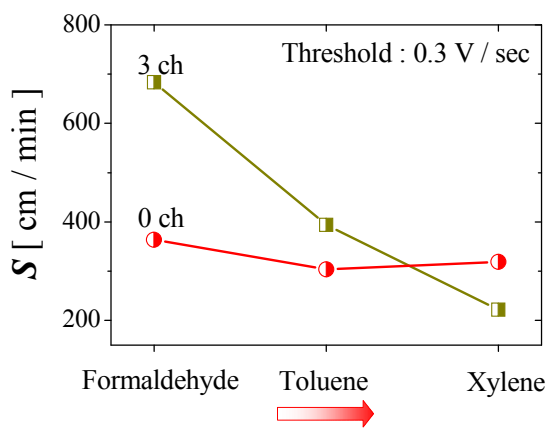

Figure 12: The $s$-values of $\operatorname{ch} 0$ and 3 sensors for the three pollutants examined, namely formaldehyde, toluene and xylene.

larger. It takes more time to diffuse as the weight is larger. However, it largely depends on the threshold value $V_{t h}$. It is important to decide the value. In general, there is a high risk of developing sick building syndrome at ceiling height.

\section{Conclusions}

It is necessary to decrease the production volume of chemical materials and the application as much as we can. Action with careful consideration to environmental risk is required. It is, however, difficult to eliminate all of the chemicals in a living environment. Many kinds of chemicals accumulate on the earth and also in indoor environments. The chemicals are decomposed and absorbed outdoors by various kinds of microbes and plants, and the concentration is comparatively lower. Meanwhile, they accumulate and reach an arbitrary saturating value in an indoor environment. The value varies from room to room environments. When a chemical occurs in an indoor environment, it is necessary to understand the concentration and its speed in every position as an aspect of risk management. In particular, the saturating value and the time are important.

In this paper, experiments to understand the diffusion characteristics of formaldehyde, toluene and xylene in an indoor environment were carried out. On the whole, when a VOC was generated it became obvious that the concentration near ceiling height, directly over the source, was higher. This result is the same as the one for smoke. The detecting speed $s$ was defined and it was derived. The sensor output is significantly influenced even by slight wind. The $s$-value becomes larger near the ceiling, but it varies considerably with wind velocity in the room.

In the future, an analysis of the polluting sources, such as curtains and carpets, will be carried out. 


\section{Acknowledgement}

A part of this investigation was supported by Grant-in-aid for Scientific (B) (No. 18310062, 2006) of Japan. We would like to express our appreciation to the Institute.

\section{References}

[1] J. Glynn Henry, Gary W. Heinke, Environmental Science and Engineering, Prentice-Hall International, New Jersey, 1996.

[2] Tunga Salthammer, Organic Indoor Air Pollutants, Wiley-VCH, Germany, 1999.

[3] Virginia I. Lohr, Caroline H. Pearson-Mims, Physical Discomfort May Be Reduced in the Presence of Interior Plants, Hort Technology, Vol.10, No.1, pp.53-58, 2000.

[4] S. Tanabe, R. Shirai, M. Manno, Y. Asai, Indoor Chemical Pollution and Emission Rates Investigated In a Real Sized Model Room, Proceedings Indoor Air 2002, No.208-213, 2002.

[5] Ayako Sawada, Takashi Oyabu, Li-mei Chen, Norihito Hirai and Katsura Izui: "Purification capability of tobacco transformed with enzymes from a methylotrophic bacterium for formaldehyde", International Journal of Phytoremediation, Vol.9, No.6, pp.487-496 (2007)

[6] Ayako Sawada, Ryuzoh Ikeda, Eiichi Tamiya, Taketoshi Yoshida, Takashi Oyabu and Hidehito Nanto: "A Novel Formaldehyde-degrading Fungus, Trichoderma virens: Isolation and Some Properties", IEICE TRANSACTIONS on Electronics, Vol.E89-C, No.12, pp.1786-1791 (2006)

[7] Ayako Sawada, Tsubasa Higashino, Takashi Oyabu, Yoshinori Takei, Hidehito Nanto, Kiyoshi Toko, Gas sensor characteristics for smoldering fire caused by a cigarette smoke, Sensors \& Actuators B, Vol.130, pp. 8893, 2008. 